\title{
Dasatinib-induced diffuse alveolar hemorrhage
}

\author{
Dhara Dave MD, John Kimbugwe MD, Randa Hazam MD, Saria Tasnim MD, Manish Patel MPH, MD
}

\begin{abstract}
The BCR-ABL tyrosine kinase inhibitor dasatinib is a potent treatment for chronic myeloid leukemia (CML). However, it is associated with pulmonary toxicities. Commonly reported dasatinib related pulmonary toxicities include pleural effusion, lung parenchymal abnormalities, and pulmonary hypertension. Diffuse alveolar hemorrhage (DAH) during treatment with dasatinib is very rare. To the best of our knowledge there are only two cases reported. Here we report a 57-year-old Caucasian woman who developed acute hypoxic respiratory failure while on dasatinib for treatment of CML. She was diagnosed with DAH suspected to be secondary to dasatinib, after other common etiologies were ruled out. There was full recovery after stopping dasatinib and treatment with corticosteroids.
\end{abstract}

Keywords: Dasatinib, pulmonary toxicity, diffuse alveolar hemorrhage, chronic myeloid leukemia

\section{INTRODUCTION}

Since the approval of imatinib for the treatment of chronic myeloid leukemia (CML), BCR-ABL tyrosine kinase inhibitors (TKIs) have drastically improved the prognosis of patients with CML. Nilotinib and dasatinib are second generation TKIs that are superior to imatinib in achieving molecular responses in $\mathrm{CML}$ patients. ${ }^{1}$ Although generally well tolerated, dasatinib's adverse effects include cytopenias, fluid retention, gastrointestinal bleeding, intracranial bleeding, and pulmonary side effects. ${ }^{2}$ The most commonly reported pulmonary toxicities include pleural effusions, lung parenchymal abnormalities, and pulmonary hypertension. ${ }^{3,4}$ Diffuse alveolar hemorrhage (DAH) during treatment with dasatinib is very rare. A literature search retrieved only two cases, and here we report the third case of a patient who developed DAH while on dasatinib.

Corresponding author: Dhara Dave Contact Information: Dhara.Dave@ttuhsc.edu DOI: $10.12746 /$ swrccc.v9i41.897

\section{CASE}

A 57-year-old Caucasian woman was hospitalized following cardiac arrest due to acute hypoxic respiratory failure. Her past medical history was significant for chronic diastolic heart failure, chronic myeloid leukemia, chronic obstructive pulmonary disease on home oxygen, stage III chronic kidney disease, obstructive sleep apnea, and pulmonary hypertension. Home medications included albuterol, tiotropium, carvedilol, losartan, furosemide, and dasatinib. The patient was switched from imatinib to dasatinib 6 weeks prior. She quit smoking 12 years ago. Two days before admission she went to the emergency department for dyspnea and dry cough. She was discharged home on levofloxacin, but continued to have worsening dyspnea. Her husband called EMS, and upon arrival of EMS, she was in respiratory failure requiring intubation at the scene. In route to the hospital, she had two cardiac arrests (non-shockable rhythm) and was resuscitated adequately.

She required high ventilator settings $\left(\mathrm{FiO}_{2} 100 \%\right.$, PEEP $10 \mathrm{~cm} \mathrm{H}_{2} \mathrm{O}$, tidal volume $380 \mathrm{ml}$ ) to maintain oxygen saturation above $89 \%$. Chest auscultation 


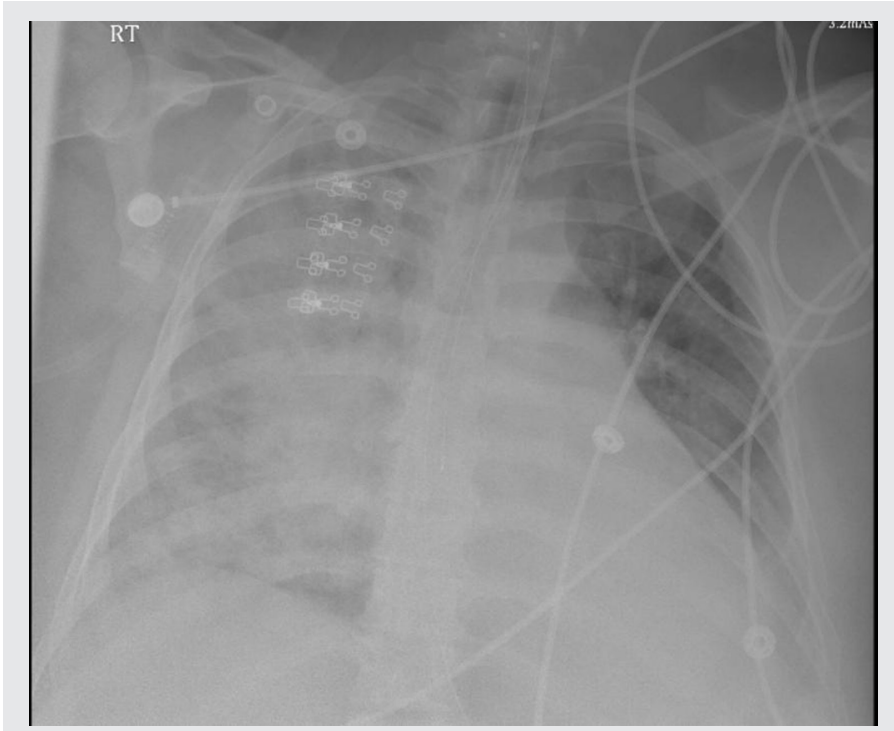

Figure 1. Portable chest x-ray revealing dense alveolar infiltrates, especially in the right lung.

revealed diffuse coarse crackles bilaterally and normal heart sounds. She had no pedal edema.

Blood work on admission showed white cell count 8200 cells $/ \mu \mathrm{L}$, hemoglobin $11 \mathrm{gm} / \mathrm{dL}$, platelets $193000 / \mu \mathrm{L}$, and procalcitonin $0.730 \mathrm{ng} / \mathrm{mL}$; her prothrombin time was 12.3 seconds (INR $=1.18$ ). During the first three days of admission, her hemoglobin count dropped to $8.8 \mathrm{gm} / \mathrm{dL}$. She had no sign of active bleeding. Her electrocardiogram showed a sinus rhythm and incomplete right bundle branch block with non-specific ST-T wave changes in anterior leads. Chest $x$-ray on admission showed extensive opacity in the right lung and a dense opacity in the left lung base. The next day the chest x-ray had worsened with bilateral opacities (Figure 1). Chest computed tomography scan showed bilateral pleural effusions with bilateral pulmonary infiltrates (Figure 2). The patient was initially placed on empiric antibiotics for hospital-acquired pneumonia; however, no clinical improvement was noted over 48 hours, and antibiotics were discontinued.

Bronchoscopy showed bloody secretions in all major, lobar, and segmental bronchi, and the consecutive aliquots of lavage fluid became bloodier, findings consistent with alveolar hemorrhage. Pathology of the bronchoalveolar lavage (BAL) sample showed

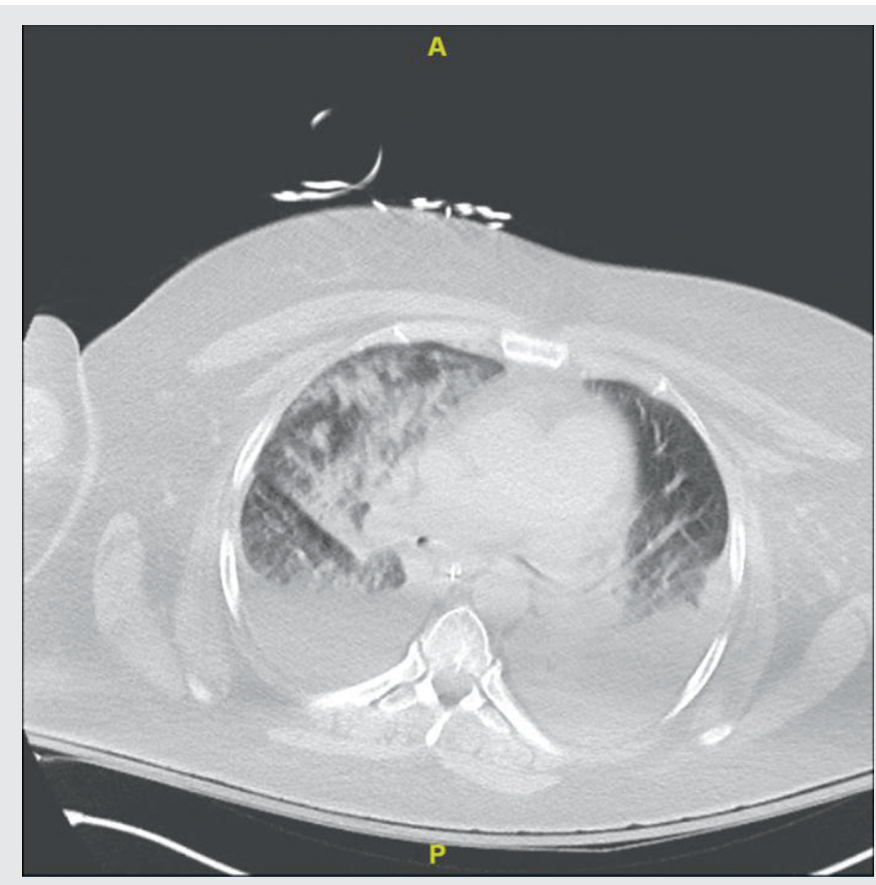

Figure 2. Computed tomography of the chest reveals bilateral pleural effusions and infiltrates, especially in the right lung.

abundant benign macrophages superimposed on acute and chronic inflammatory cells and red blood cells; no malignant cells were identified. Blood and BAL cultures were negative. Antinuclear antibodies (ANA), cytoplasmic antineutrophil cytoplasmic antibodies (c-ANCA), perinuclear antineutrophil cytoplasmic antibodies ( $p-A N C A)$, and anti-glomerular basement membrane antibodies (anti GBM) were negative. The diagnosis of dasatinib-induced DAH was made, and the drug was stopped. She was started on high dose methylprednisolone for 7 days. Her condition progressively improved, and she was extubated successfully on day 7 (Figure 3). She had an uneventful course afterward, was transferred to the medical floor, and later was discharged home on her baseline oxygen requirement and tapering dose of corticosteroids.

\section{Discussion}

Dasatinib is a highly effective second-generation TKI approved for the treatment of imatinib-resistant or intolerant chronic myeloid leukemia and 


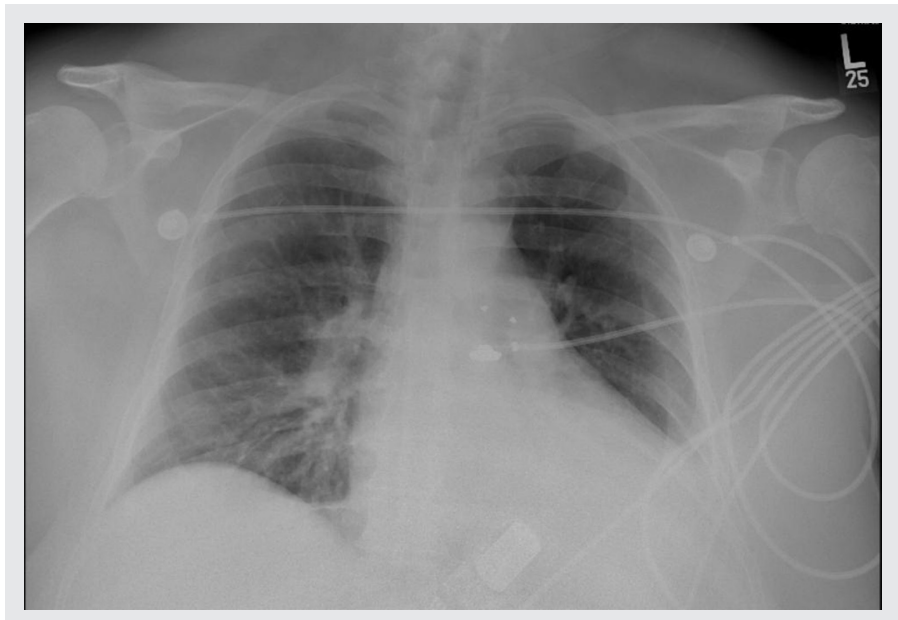

Figure 3. Portable chest x-ray reveals significant clearing of alveolar infiltrates.

Philadelphia-positive acute lymphoblastic leukemia. The activity of dasatinib is 325 times that of imatinib, and it is an important drug for the treatment of CML. ${ }^{1}$ The most common reported pulmonary toxicities are pleural effusion, lung parenchymal abnormalities, and pulmonary arterial hypertension, and these can occur simultaneously or separately. ${ }^{3,4}$

In clinical trials, the incidence of dasatinib-induced pleural effusion ranged from $10 \%$ to over $30 \%$ and was dose-dependent. ${ }^{3,5}$ Pulmonary arterial hypertension is not as common with only about $5 \%$ incidence noted in the DASISION trial, and it is usually reversible following discontinuation of dasatinib. ${ }^{6}$ Pneumonitis or lung parenchymal abnormalities are very rare and reported in only nine of 40 patients in a series reported by Bergeron et al. ${ }^{4}$ Of these nine patients, eight improved with stopping dasatinib, and one patient was placed on glucocorticoid therapy while continuing dasatinib. Seven of them had full recovery, and two had partial recovery. ${ }^{4}$

Diffuse alveolar hemorrhage is an extremely rare manifestation of dasatinib-related pulmonary toxicity with only two reported cases to date. ${ }^{7,8}$ The first case presented at a meeting in Japan in 2009 had pulmonary and urinary bleeding while on dasatinib. ${ }^{7}$ The second case was a 39-year-old man who presented with hemoptysis, cough, and dyspnea while on dasatinib. ${ }^{8}$ Chest CT scan showed ground glass opacities. Bronchoscopy and BAL were not possible due to severe hypoxemia. Dasatinib-induced alveolar hemorrhage was suspected based on clinical findings and the observation of intratracheal bleeding during endotracheal intubation. The patient's condition improved immediately after dasatinib withdrawal and initiation of corticosteroids therapy. The authors concluded that dasatinib was the cause of alveolar hemorrhage.

In our case the increasing airspace disease on imaging and the decrease in hemoglobin raised suspicion for pulmonary bleeding. Bronchoscopy confirmed alveolar hemorrhage since each consecutive BAL aliquots were bloodier than the previous sample and there were no obvious endobronchial lesions. Vasculitis and infection were ruled out. Pulmonary edema could not explain the hemorrhage given the bloodier returning aliquots during $\mathrm{BAL}$ and the increasing infiltrates and dropping hemoglobin. Repeat echocardiogram was unchanged from her baseline. After reviewing literature, we considered the possibility of dasatinib-induced alveolar hemorrhage. Furthermore, none of her other medications has been associated with DAH. Her respiratory function improved after dasatinib was stopped and with treatment with corticosteroids.

The mechanism of dasatinib-induced DAH is uncertain. Sakoda et al. suggested alveolar epithelial and interstitial damage as KL-6 (sialylated carbohydrate antigen) and SP-D (surfactant protein-D) levels were elevated in their case. ${ }^{8}$ Dasatinib has been reported to cause thrombocytopenia and bleeding in several organs. However, the lowest platelet count in our case was $115,000 / \mu \mathrm{L}$, and so thrombocytopenia was unlikely the cause. Due to the scarcity of data linking dasatinib to $\mathrm{DAH}$, no cause-effect relationship can be established at present. However, besides the absence of other potential etiologies, clinical association of dasatinib with DAH has been observed in the two reported cases and our present case. In our case the onset of DAH was after 6 weeks of dasatinib treatment; the other case was after one year. ${ }^{8}$ Therefore, it is difficult to establish a time frame for this complication.

Dasatinib has been used with increasing frequency, and even though it is well tolerated, it is important to think of its pulmonary toxicities, especially in patients with a deteriorating respiratory status with no 
other obvious causes. Therefore, clinicians should be aware that dasatinib can cause rare but significant alveolar hemorrhage. This case should encourage more reporting of similar cases to raise awareness of this rare toxicity.

Article citation: Dave D, Kimbugwe J, Hazam R, Tasnim S, Patel M. Dasatinib-induced diffuse alveolar hemorrhage. The Southwest Respiratory and Critical Care Chronicles 2021;9(41):54-57

From: Department of Internal Medicine, Texas Tech University Health Sciences Center, Amarillo, Texas

Submitted: $7 / 7 / 2021$

Accepted: 9/30/2021

Reviewer: Kenneth Nugent MD

Conflicts of interest: none

This work is licensed under a Creative Commons Attribution-ShareAlike 4.0 International License.

\section{REFERENCES}

1. O'Hare T, Walters DK, Stoffregen EP, et al. In vitro activity of Bcr-Abl inhibitors AMN107 and BMS-354825 against clinically relevant imatinib-resistant $\mathrm{Abl}$ kinase domain mutants. Cancer Res 2005 Jun 1;65(11):4500-5.

2. Fox LC, Cummins KD, Costello B, et al. The incidence and natural history of dasatinib complications in the treatment of chronic myeloid leukemia. Blood Adv 2017 May 15;1(13): 802-811.

3. Conchon M, Freitas CM, Rego MA, et al. Dasatinib-clinical trials and management of adverse events in imatinib resistant/intolerant chronic myeloid leukemia. Rev Bras Hematol Hemoter 2011;33(2):131-9.

4. Bergeron A, Réa D, Levy V, et al. Lung abnormalities after dasatinib treatment for chronic myeloid leukemia: a case series. Am J Respir Crit Care Med 2007 Oct 15;176(8):814-8.

5. Brixey AG, Light RW. Pleural effusions due to dasatinib. Curr Opin Pulm Med 2010 Jul;16(4):351-6.

6. Cortes JE, Saglio G, Kantarjian HM, et al. Final 5-year study results of DASISION: the dasatinib versus imatinib study in treatment-naïve chronic myeloid leukemia patients trial. J Clin Oncol 2016 Jul 10;34(20):2333-40.

7. Nishikawa A, Fujiwara S, Hatano K, et al. A case of pulmonary and urinary bleedings caused by dasatinib in posttransplant recurrent ph-ALL. Jpn J Clin Hematol 50:1168, 2009 (in Japanese).

8. Sakoda Y, Arimori Y, Ueno M, et al. A Suspected case of an alveolar haemorrhage caused by dasatinib. Intern Med 2017;56(2):203-206. 